\title{
miR-491-3p is Downregulated in Retinoblastoma and Inhibit Tumor Cells Growth and Metastasis by Targeting SNN
}

\author{
Yang Hu${ }^{1}$ (D) $\cdot$ Ming Zhao ${ }^{1} \cdot \mathrm{Li} \mathrm{Li}^{1} \cdot$ Jie Ding ${ }^{1} \cdot$ Yu-Min Gui ${ }^{1} \cdot$ Tan-Wei Wei $^{1}$
}

Received: 30 April 2020 / Accepted: 29 September 2020 / Published online: 23 October 2020

(c) The Author(s) 2020

\begin{abstract}
Retinoblastoma $(\mathrm{Rb})$ is the most common pediatric malignant tumor of the eyes. Previous studies demonstrated that miR-491-3p is downregulated in various cancers. However, its function in $\mathrm{Rb}$ remains unknown. A total of 15 pairs of primary Rb tissues and adjacent noncancerous tissues were collected. Quantitative real-time PCR (qRT-PCR) was used to investigate the expression profiles of miR-491-3p. qRTPCR, western blotting and in situ immunocytochemistry were performed to investigate the expression profiles of epithelial-mesenchymal transition-related proteins (E-cadherin, Vimentin and $\mathrm{N}$-cadherin) in $\mathrm{Rb}$ tissues and $\mathrm{Rb}$ cell lines as well as cell morphology. Cell proliferation was estimated by MTS and colony formation assays. Apoptosis was determined by FACS, cell migration and invasion were analyzed using transwell chambers. MiR-491-3p's target genes were predicted using target gene prediction databases. The interplay between miR-491-3p and SNN was evaluated through dual luciferase reporter gene assay. MiR-491-3p was significantly downregulated in mixed collection of 15 pairs of $\mathrm{Rb}$ tissues and $\mathrm{Rb}$ cell lines. Overexpression of miR-491-3p enhanced apoptosis, and significantly suppressed proliferation, migration and invasion of Rb cells. In contrast, the present of miR-491-3p inhibitor showed reversed results which apoptosis decreased, while cell proliferation of ARPE-19 cells increased. In addition, miR-491-3p increased the expression of E-cadherin, and dramatically decreased the expression of Vimentin and N-cadherin in $\mathrm{Rb}$ tissues and $\mathrm{Rb}$ cell lines, noticeable changes in morphology, too, as cells became less cohesive and more adhering. We found out that SNN was the pairing target of miR-491-3p and result showed that miR-491-3p and SNN interacted with each other. We also found out that the effects of miR-491-3p were in Rb cells were almost entirely canceled out at the overexpression of SNN. Our findings collectively suggest that miR-491-3p is an important tumor suppressor in Rb, which inhibits
\end{abstract}

Electronic supplementary material The online version of this article (https://doi.org/10.1007/s1052 8-020-10007-w) contains supplementary material, which is available to authorized users.

Extended author information available on the last page of the article 
tumor growth and metastasis in $\mathrm{Rb}$. These implicate it may be explored as a new therapeutic target in $\mathrm{Rb}$.

Keywords Retinoblastoma $\cdot \mathrm{miR}-491-3 \mathrm{p} \cdot$ Tumor growth $\cdot$ Metastasis $\cdot$ Epithelialmesenchymal transition

$\begin{array}{ll}\text { Abbreviations } \\ \mathrm{Rb} & \text { Retinoblastoma } \\ \text { miRNAs } & \text { MicroRNAs } \\ \mathrm{HRM} & \text { Hypoxia-regulated miRNA } \\ \text { EMT } & \text { Epithelial-mesenchymal transition } \\ \text { ICRB } & \text { International Classification of Retinoblastoma } \\ \mathrm{NC} & \text { Negative control } \\ \mathrm{SNN} & \text { Stannin } \\ \text { cDNA } & \text { Complementary DNA } \\ \text { RIPA } & \text { Radioimmunoprecipitation assay } \\ \text { PVDF } & \text { Polyvinylidene difluoride membranes } \\ \text { ANOVA } & \text { Analysis of variance }\end{array}$

\section{Introduction}

Retinoblastoma $(\mathrm{Rb})$ is a form of cancer that rapidly develops from the immature cells of a retina, the light-detecting tissue of the eye. It is the most common primary malignant intraocular cancer in children, and it is almost exclusively found in young children (Neoplasms of the Eye 2003). The morbidity rate of Rb is about 1:15,000-1:20,000 in human infant (0-5 years). It is estimated that almost 9,000 new cases are annually reported worldwide (Ramirez-Ortiz et al. 2017). The Rb growth usually occurs under the retina and toward the vitreous cavity, result in the retinal detachment. In addition, it can spread to the central nervous system, and causes the death of patient (Fernandes et al. 2017). Hence, there is a dire need to explore the initiation and progression of $\mathrm{Rb}$ as well as its underlying mechanisms, which is helpful to develop more effective treatments.

MicroRNAs (miRNAs) are small noncoding single stranded RNAs and they regulate cellular and molecular processes, particularly gene expression by binding to complementary sequences in the $3^{\prime}$-untranslated region (3'-UTR) of target mRNAs. Through their transcriptional regulatory functions, miRNAs can control tumor proliferation, invasion and metastasis (Sethi et al. 2014a). Irregular expressions of miRNAs often contribute to the initiation and progression of cancers, for instance, Zhu et al. has demonstrated that miR-17-5p was able to enhance pancreatic cancer proliferation (Zhu et al. 2018), while Gao et al.'s study suggested that miRNA-223 may also play an essential part in both hematological malignancies and solid tumors (Gao et al. 2017). Extensive literatures also demonstrated that miRNAs play an important role in Rb. Researchers have found that miR-18a could inhibit tumorigenic potential of $\mathrm{Rb}$ while inhibition of miR-106b significantly increased 
the expression of the Rb genes (Busch et al. 2017; Samal et al. 2017). Additionally, miR-21 inhibitor suppressed the progression of $\mathrm{Rb}$ via the modulation of PTEN/ PI3K/AKT pathway (Gui et al. 2016). In a previous literature, miR-491-3p was identified as a hypoxia-regulated miRNA (HRM) in Rb cells using microarray analysis (Xu et al. 2011). More importantly, miR-491-3p was already found playing a critical role in various cancers. In tongue cancer, mTORC2 downregulated miR-491-3p expression by inactivating FOXO1 (Zheng et al. 2015); while in osteosarcoma miR491-3p functions as a tumor suppressor to attenuate the potential of growth and invasion by targeting TSPAN1 (Duan et al. 2017). Other researchers also found that miR-491-3p was able to attenuate multidrug resistance of hepatocellular carcinoma (Zhao et al. 2017). However, the biological role of miR-491-3p in Rb development remains elusive. EMT is a process by which epithelial cells lose their cell polarity and cell-cell adhesion and gain migratory and invasive properties to become mesenchymal stem cells; and it is a hallmark of initiation of metastasis in cancer progression. Using western blotting analysis and immunocytochemistry, we evaluated the expression levels of three EMT biomarkers: E-cadherin, Vimentin and N-cadherin, as well as morphology changes in Rb cells. Among them, E-cadherin is an epithelial cell marker, while Vimentin and N-cadherin are mesenchymal cell markers (Zeisberg and Neilson 2009).

Stannin (SNN) is a highly conserved vertebrate protein that consists of a single transmembrane helix, an unstructured linker domain, and a cytoplasmic domain. Previous study has revealed that it is closely linked to trimethyltin (TMT) toxicity and has played important role in cell apoptosis (Reese et al. 2005). Numerous studies have also indicated SNN plays the important role in tumor's growth cycle (Reese et al. 2006). However, there is no study to examine the interaction between SNN and miR-491-3p and how this interaction can regulate the progression of tumor.

In this study, we first examined amount of miR-491-3p expression in $\mathrm{Rb}$ tissues and cells compared with normal, noncancerous tissues. Subsequently, we investigated the role of miR-491-3p in Rb cell proliferation, apoptosis, migration and invasion. Last, we used EMT (epithelial-mesenchymal transition) markers to test the role of miR-491-3p in $\mathrm{Rb}$ metastasis. Our results demonstrated the importance of miR-491-3p in regulating $\mathrm{Rb}$ development. This study was also the first to reveal miR-491-3p and SNN's interaction to regulate proliferation, migration, invasion and apoptosis of $\mathrm{Rb}$ cell. This study provided novel insights into the biological function of miR-491-3p and showed that it may be a potential therapeutic target for $\mathrm{Rb}$.

\section{Materials and Methods}

\section{Tissue Samples}

A total of 15 pairs of primary Rb tissues and adjacent noncancerous tissues were collected from the Wuhan University of Science and Technology Affiliated Hospital, Puren Hospital (Wuhan, China) between 2016 and 2017. The median age of patients was 2.5 years. Table 1 showed detailed information about the patients' subphenotype, gender, age, tumor size, tumor stage and whether the patient has family 
Table 1 The count of clinical characteristics in retinoblastoma

\begin{tabular}{ll}
\hline Subphenotypes & Case $(N=15)$ \\
\hline Gender & \\
Male & 8 \\
Female & 7 \\
Age (year) & \\
$\leq 5$ & 9 \\
$>5$ & 6 \\
Tumor size (mm) & \\
$\leq 15$ & 7 \\
$>15$ & 8 \\
Tumor stage & \\
I+II & 4 \\
III + IV & 11 \\
Family history & \\
Positive & 10 \\
Negative & 5 \\
\hline
\end{tabular}

history of retinoblastoma. The obtained tissues were immediately snap-frozen in liquid nitrogen and stored at $-80{ }^{\circ} \mathrm{C}$. The diagnosis of $\mathrm{Rb}$ was confirmed by histopathological examination. All the cases were diagnosed and classified according to the International Classification of Retinoblastoma (ICRB). None of the subjects received any biotherapy, chemotherapy or radiotherapy treatment before recruitment to this study. All experiments were preformed according to the university's human object use guidelines with approval from IRB (institution review board) and the hospital's ethics committee. Signed informed consent from the participants or parent/ legal guardian was obtained before the experiment.

\section{Cell Lines and Cell Culture}

Human retinal pigment epithelium cell line ARPE-19 and Rb cell lines (Weri-Rb1 and Y79) were purchased from the American Type Culture Collection (Manassas, VA, USA). ARPE-19 cells were cultured in a 1:1 mixture of Dulbecco's modified Eagle's medium (DMEM) and Ham's F-12 medium (Invitrogen, Carlsbad, CA), supplemented with $10 \%$ fetal bovine serum (FBS, Omega Scientific, Tarzana, CA), $1 \%$ penicillin-streptomycin and $2 \mathrm{mM}$ glutamine (Invitrogen, Carlsbad, CA), whereas Weri-Rb1 and Y79 cells were cultured in RPMI-1640 medium with 20\% FBS, 1\% penicillin-streptomycin and $2 \mathrm{mM}$ glutamine (Invitrogen, Carlsbad, CA). All cell lines were cultured at $37{ }^{\circ} \mathrm{C}$ with $5 \% \mathrm{CO}_{2}$.

\section{Cell Transfection}

The miR-491-3p mimics (5'-AGTAGAAGGGAATCTTGCATAAG-3') and its negative control (mimics NC) (5'-ACAGAGCTATAGATGTAAGTGAG-3') were 
purchased from GenePharma Co., Ltd (Shanghai, China). miR-491-3p inhibitor (5'CUUAUGCAAGAUUCCCUUCUAC-3') and its negative control (inhibitor NC) ('5-GGUUCGUACGUACACUGUUCA-3') were purchased from Sigma-Aldrich. Cells were transfected with miR-491-3p mimics, mimics NC, miR-491-3p inhibitor or inhibitor NC using Lipofectamine 2000 reagent (Invitrogen; Thermo Fisher Scientific, Inc.) according to the manufacturer instructions. Briefly, $5 \mu \mathrm{L}$ Lipofectamine 2000 combined with miRNAs at a concentration of $30 \mathrm{nmol} / \mathrm{L}$ were incubated in Opti-MEM I reduced medium (Invitrogen) for $20 \mathrm{~min}$ prior to transfection. The mixture was then added into a 6 -well plate with $5 \times 10^{4}$ cells per well. Medium were changed to complete growth medium with $20 \%$ FBS after $6 \mathrm{~h}$ incubation at $37^{\circ} \mathrm{C}$. After transfection for $48 \mathrm{~h}$, cells were harvested. Stannin (SNN) human tagged ORF clone lentiviral particle (oe-SNN), Lenti-ORF control particles (vector) and transfection reagent TurboFectin were obtained from OriGene and followed manufacturer's preparation protocols.

\section{RNA Isolation and Quantitative Real-Time PCR (qRT-PCR)}

Total RNAs was isolated by using a TRIzol Reagent (Invitrogen, Carlsbad, CA) according to the manufacturer's instructions. The extracted RNA concentration and quantity were assayed using the NanoDrop ND-1000 Spectrophotometer (NanoDrop, Wilmington, DE). The RNA samples were reverse transcribed into complementary DNA (cDNA) using the TaqMan ${ }^{\mathrm{TM}}$ MicroRNA Reverse Transcription Kit (Applied Biosystems, Foster City, USA) following the manufacturer's protocols. Quantitative real-time PCR (qRT-PCR) was executed by TaqMan Universal PCR Master Mix (Applied Biosystems, Foster City, USA). The amplification was monitored on the 7500 Fast Real-Time PCR System (Applied Biosystems, Foster City, USA), and the thermal cycling conditions were $95{ }^{\circ} \mathrm{C}$ for $5 \mathrm{~min}$, followed by 40 cycles of $95{ }^{\circ} \mathrm{C}$ for $10 \mathrm{~s}$ and $60{ }^{\circ} \mathrm{C}$ for $45 \mathrm{~s}$. U6 was used as an internal control. The following primers were used for the qRT-PCR: miR-491-3p forward primer, $5^{\prime}$-ATG CAAGATTCCCTTCTACAAA-3' and reverse primer, 5'-CATGATCAGCTGGGC CAAGA-3'; U6 forward primer, 5'-CTCGCTTCGGCAGCACA-3' and reverse primer, 5'-AACGCTTCACGAATTTGCGT-3', SNN forward primer, 5'-AGTCAG AGGACGAGGAGAGCAT-3' and reverse primer, 5'-ATCCTGGCTCAGCCGTGG ACT-3'. Relative expression levels were evaluated using the $2^{-\Delta \Delta C_{\mathrm{t}}}$ method. All the reactions were performed in triplicate.

\section{MTS Assay}

The proliferation of the transfected cells in each group was determined by using the MTS solution cell proliferation assay kits (Promega Corporation, WI, USA) according to the manufacturer's instructions. The MTS assays were performed on the four consecutive time points ( $0 \mathrm{~h}, 24 \mathrm{~h}, 48 \mathrm{~h}$ and $72 \mathrm{~h})$. The absorbance at $490 \mathrm{~nm}$ was read by SpectraMax M2 microplate reader (Molecular Devices, LLC, CA, USA), and then the cell proliferation curve was established. The experiment was independently executed three times. 


\section{Soft Agar Colony Formation Assay}

Transfected cells were prepared as a single cell suspension in complete DMEM media, and mixed with $0.6 \%$ (equal volume) low-melting point agarose (SigmaAldrich Co., St Louis, MO, USA). The mixture was laid on top of $0.6 \%$ solidified agarose in DMEM in 6-well plates $\left(1 \times 10^{3}\right.$ cells/well). The growth medium was changed regularly every 3-4 days for 2 weeks. Cell colonies containing at least 50 cells were stained with crystal violet (Sigma-Aldrich Co., St Louis, MO, USA) and counted. The images were captured under a microscope (Leica Microsystems, Wetzlar, Germany) and analyzed. The experiment was performed in triplicate, and independently performed three times.

\section{Apoptosis Assay}

Cell apoptosis was performed using the FITC-Annexin V Apoptosis Detection Kit I (BestBio, Shanghai, China) according to the manufacturer's instructions. Briefly, the transfected Weri-Rb1 and Y79 Cells were resuspended at a density of $1 \times 10^{6}$ cells/mL in PBS, then stained with FITC-Annexin V and propidium iodide (PI) for 15 min. The cells were analyzed using a cytoflex flow cytometer and Beckman CXP software (Beckman Coulter, Pasadena, CA, USA).

\section{Cell Migration and Invasion Assays}

The cell migration and invasion assays were performed using the $8 \mu \mathrm{m}$ pore size chamber inserts containing polyethylene terephthalate membranes (Corning Incorporated, Corning, NY, USA). For invasion assay, the transwell chambers were precoated with $30 \mu \mathrm{L} \mathrm{20 \%} \mathrm{Matrigel} \mathrm{(BD} \mathrm{Biosciences,} \mathrm{Franklin} \mathrm{Lakes,} \mathrm{NJ,} \mathrm{USA)}$ diluted in RPMI-1640 medium. A volume of $100 \mu \mathrm{L}$ cells $\left(0.5 \times 10^{6}\right.$ cells $\left./ \mathrm{mL}\right)$ was resuspended in $200 \mu \mathrm{L}$ RPMI-1640 medium without FBS, and seeded in the upper compartment of the chamber. In addition, $500 \mu \mathrm{L}$ medium with $10 \%$ FBS was added into the lower compartment of the chamber. Following $24 \mathrm{~h}$ incubation, the cells attached on the upper surface were removed using cotton swabs. The migrated and invaded cells were counted and images were taken under an inverted microscope in five randomly selected visual fields (Leica Microsystems, Wetzlar, Germany). Both of the assays were repeated independently three times.

\section{Western Blotting}

The protein was extracted from $\mathrm{Rb}$ tissues (from mixture of $15 \mathrm{Rb}$ patients) and transfected Weri-Rb1 and Y79 cells using Radioimmunoprecipitation assay (RIPA) lysis buffer (Auragene Bioscience Co., Changsha, China) according to the manufacturer's protocols. The concentration of extracted proteins were determined by Bradford protein assay reagent (Beyotime Biotechnology, Suzhou, China).

The proteins were subjected to $12 \%$ SDS-PAGE (Bioworld Technology, Inc., St. Louis Park, MN, USA) and transferred to polyvinylidene difluoride membranes 
(PVDF) (Millipore, Bedford, MA, USA). The membranes were blocked with 5\% nonfat milk in TBST buffer (Tris-buffered saline plus $0.05 \%$ Tween-20) for $2 \mathrm{~h}$ at room temperature, and then incubated with primary antibodies (E-cadherin (1:1500, ab15148, Abcam, China), Vimentin (1:1000, ab24525, Abcam, China), N-cadherin (1:2500, ab76057, Abcam, China), SNN (1:1000, ab121330, Abcam, China), GAPDH (1:5000, ab8245, Abcam, China)) overnight at $4{ }^{\circ} \mathrm{C}$, washed three times with TBST and then incubated with a suitable secondary antibody [horseradish peroxidase-conjugated secondary antibody (ab7090, Abcam, China)] at room temperature. The membranes were visualized with enhanced chemiluminescence solution (Pierce, Thermo Fisher Scientific, Rockford, IL, USA) according to the manufacturer's instructions. Images were captured using the FluorChem imaging system (Alpha Innotech, San Leandro, CA, USA), and densitometry analysis was performed using the Image J software. GAPDH was used as loading control.

\section{miRNA Target Prediction}

Four publicly available databases were used for miRNA target prediction including Hoctar, miRDB, mirDIP and Targetscan. The prediction criteria were first 10th percentile of the ranked list for Hoctar (Gennarino et al. 2011), target score higher than 80 for miRDB (Wong and Wang 2015), "very high" and integrated score of higher than 0.5 for mirDIP (Tokar et al. 2018) and threshold was set to cumulative weighted context ++ score less than -0.4 for Targetscan (Ma et al. 2018). The final prediction result was taken where these four prediction tools intersected. The result of the final prediction then be confirmed with luciferase reporter assay.

\section{Luciferase Reporter Assay}

The full length 3'-UTR of SNN and the mutant derivative devoid of the miR-491-3p target site were amplified and cloned into psi-CHECK2 luciferase reporter vector to construct SNN 3'UTR luciferase reporter (SNN-WT) and target site mutation SNN luciferase reporter (SNN-MUT) respectively. The constructed nucleotide sequences of the plasmids were confirmed by DNA sequencing technique, and transfected with miR-493-3p mimics or mimics NC by Lipofectamine 2000 transfection reagent (Invitrogen) for $48 \mathrm{~h}$. After that, luciferase reporter assays were carried out with Luciferase Reporter Assay kit (Promega) according to manufacturer protocol.

\section{In Situ Immunocytochemistry (ICC)}

Cover slips was immersed in $2 \% \mathrm{H}_{2} \mathrm{O}_{2}$ for $4 \mathrm{~h}$ and then washed 10 times with water and followed by $\mathrm{ddH}_{2} \mathrm{O}$ for 3 times. Cover slips was individually pull out and sterilized with flame, allowed to cool and placed in a container, then, coated with $100 \mu \mathrm{g} /$ $\mathrm{ml}$ sterilized Poly-L-lysine solution for 5 min and rinsed with $\mathrm{ddH}_{2} \mathrm{O}$ for 3 times. Cells were seeded for overnight to the container. Media was removed and rinsed twice with 1XPBS at room temperature, and fixed in cool $4 \%$ paraformaldehyde in 1XPBS on ice for $15 \mathrm{~min}$. After that, paraformaldehyde solution was removed and 
rinsed with 1XPBS 3 times at room temperature and the cells were permeabilized with $0.3 \%$ Triton $\mathrm{X}-100$ in PBS for $15 \mathrm{~min} .0 .3 \% \mathrm{H}_{2} \mathrm{O}_{2}$-methanol solution was added and incubated for $10 \mathrm{~min}$ after removing Triton X-100 solutions with PBS. After that, incubating solution was removed and incubated with $2 \%$ BSA in 1XPBS for $30 \mathrm{~min}$ at $37{ }^{\circ} \mathrm{C}$ to block nonspecific hybridization. Antibodies (anti-E-Cadherin; ab15148, anti-Vimentin; ab137321) were diluted in 1XPBS, 2\% BSA according to manufacturer's instruction and applied to the cells on cover slips and incubated for $1 \mathrm{~h}$ at $37^{\circ} \mathrm{C}$. After that the incubating buffer was removed and rinsed 3 times with 1XPBS, then incubated with Alexa Fluor secondary antibodies (Abcam China) in 1XPBS, $2 \% \mathrm{BSA}$ for $30 \mathrm{~min}$ at $37^{\circ} \mathrm{C}$. After the incubation, cover slips were rinsed with 1XPBS 4 times and immersed in DAB solution. Cover slips were closely monitor and rinsed with 1XPBS as soon as the cells turned brown. Cells then were counterstained in hematoxylin for $10 \mathrm{~min}$, washed in 1 XPBS, immersed in $1 \%$ acid-alcohol and rinsed with tab water. The coverslips were then washed with PBS and were mounted onto glass slides for imaging.

\section{Statistical Analyses}

All statistical analyses were performed using SPSS 18.0 software (SPSS Inc., Chicago, IL, USA). Data are represented as mean \pm standard deviation (SD), and statistical significance was set at $p<0.05$. Differences between two or multiple groups were analyzed by Student's t-tests or one-way analysis of variance (ANOVA) followed with Tukey's post-hoc analysis, respectively.

\section{Results}

\section{miR-491-3p is Downregulated and Epithelial-Mesenchymal Transition is Activated in Rb Tissues and Rb Cell Lines}

First, qRT-PCR was performed to evaluate the expression of miR-491-3p in Rb and adjacent noncancerous tissues. As shown in Fig. 1a, the expressions of miR-491-3p were significantly downregulated (almost 50\%) in Rb tissues compared to noncancerous tissues. Second, we also examined miR-491-3p expression in Rb cell lines (Weri-Rb1 and Y79), and found that miR-491-3p was significantly inhibited nearly $50 \%$ in Weri-Rb1 and Y79 cell lines compared to ARPE-19 cell line (Fig. 1b). Our study results demonstrated that miR-491-3p was downregulated in $\mathrm{Rb}$ tissues and $\mathrm{Rb}$ cell lines, which may suggest miR-491-3p may play an important role Rb development. As shown in Fig. 1c, protein expression of the E-cadherin from the $15 \mathrm{Rb}$ patients was significantly decreased in tumors, whereas $\mathrm{N}$-cadherin and Vimentin expressions were significantly increased as compared to normal ARPE-19 cell. The results indicated that miR-491-3p was downregulated and EMT was activated in Rb tissues and $\mathrm{Rb}$ cell lines. 
A

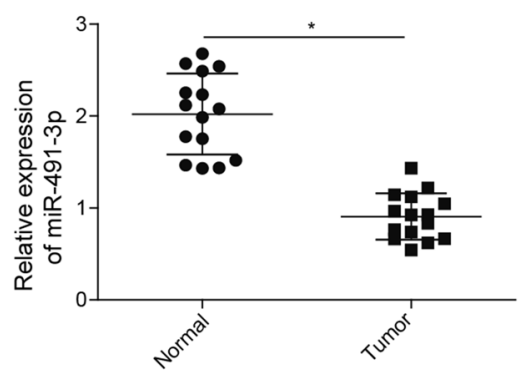

C

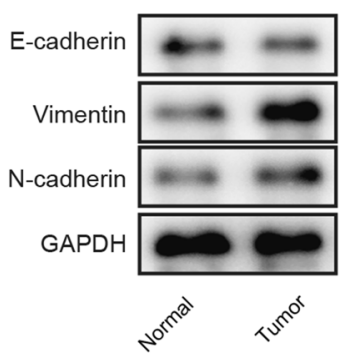

B
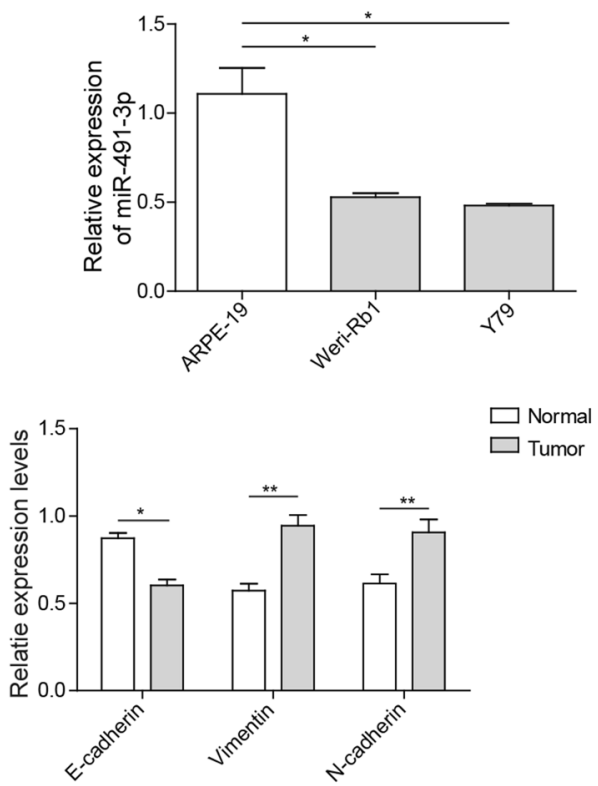

Fig. 1 Expression profiles of miR-491-3p in Rb tissues and $\mathrm{Rb}$ cell lines and epithelial-mesenchymal transition occurs. a qRT-PCR analysis of miR-491-3p expression in Rb tissues and adjacent noncancerous tissues. b qRT-PCR analysis of miR-491-3p expression in Rb cell lines (Weri-Rb1 and Y79) compared to normal human retinal pigment epithelial cell line ARPE-19. c Western blot of the expression of EMT-related proteins-E-cadherin, vimentin and N-cadherin in normal ARPE-19 or Rb tissue. All results are from three independent experiments. Bars represent mean $\pm \mathrm{SD}\left(n=3,{ }^{*} p<0.05\right)$

\section{Overexpression of miR-491-3p Suppresses Cell Proliferation of Rb Cells In Vitro}

To understand the function of miR-491-3p in cell proliferation of Rb, Y79 and Weri-Rb1 cells were transfected with either miR-491-3p mimics, mimics NC, subsequently, the proliferation activities were measured using MTS and colony formation assays. We first confirmed the effectiveness of miR-491-3p overexpression by miR-491-3p mimics as shown in Fig. 2a. The miR-491-3p levels of cells transfected with miR-491-3p mimics were about 12 times more than cells transfected with mimics NC (Fig. 2a). Further, MTS assay results demonstrated that miR-491-3p overexpression significantly suppressed cell proliferation in Y79 cells and in Weri-Rb1 cells from 24 to $72 \mathrm{~h}$ (Fig. 2b). The soft agar colony formation assay showed that colonies of both $\mathrm{Rb}$ cell lines were significantly inhibited after miR-491-3p mimics transfection compared to the colony formation of both cell lines transfected with mimics NC (Fig. 2c). The results suggested that miR-491-3p was critical for Rb cell proliferation. 


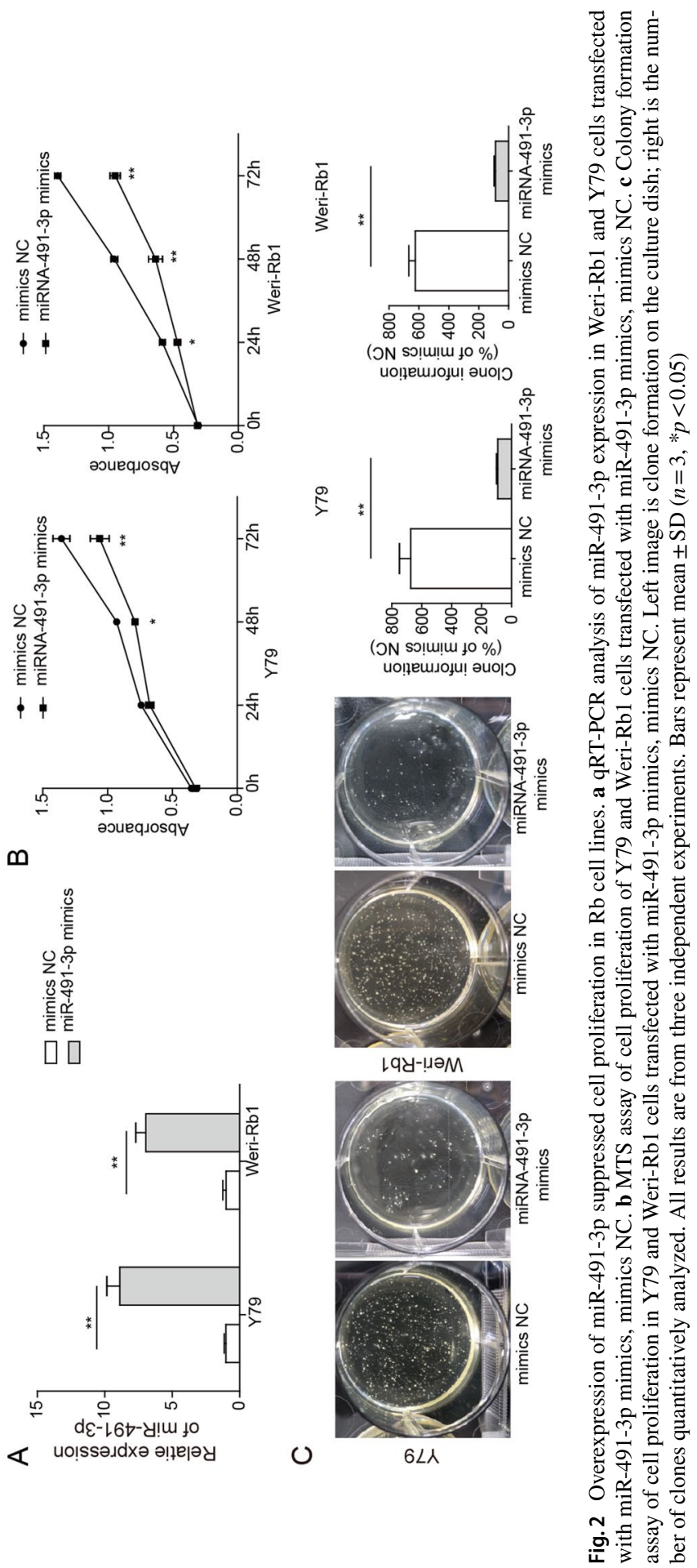




\section{Overexpression of miR-491-3p Suppresses Migration and Invasion of Rb Cells In Vitro}

We also elucidated the role of miR-491-3p in cell migration and invasion using transwell assays. After transfected with either miR-491-3p mimics or mimics $\mathrm{NC}$, the migrated cells were decreased nearly $75 \%$ for the Y79 cell line and $65 \%$ for Weri-Rb1 cell line transfected with miR-491-3p mimics as compared to cells transfected with mimics NC (Fig. 3a). Furthermore, we also found that the percentages of invaded cells were markedly decreased more than $50 \%$ in both $\mathrm{Rb}$ cell lines after transfected with miR-491-3p mimics as compared to the cells transfected miR-491-3p NC (Fig. 3b). The results suggested that overexpression of miR-491-3p reduced the migration and invasion capacity of $\mathrm{Rb}$ cells.

\section{Overexpression of miR-491-3p Enhances Cell Apoptosis and Affects EMT Process of Rb Cells In Vitro}

To further examine the role of miR-491-3p in Rb, we performed FACS assay to explore mechanism of miR-491-3p regulation of $\mathrm{Rb}$ cell growth. We found that overexpression of miR-491-3p promoted cell apoptosis in both Y79 and Weri-Rb1 cells


Fig. 3 Overexpression of miR-491-3p decreases cell migration and invasion in $\mathrm{Rb}$ cell lines. a Left image is staining of cell migration for cell transfected with miR-491-3p mimics, mimics NC under the inverted microscope (magnification, $\times 100$ ); right image is statistical analysis of left image. b Left image is staining of cell invasion for cell transfected with miR-491-3p mimics, mimics NC under the inverted microscope (magnification, $\times 100$ ); right image is statistical analysis of left image. All results are from three independent experiments. Bars represent mean $\pm \mathrm{SD}(n=3, * p<0.05)$ 
(Fig. 4a). With miR-491-3p mimics transfection, cell apoptosis increased approximately two-fold in both Y79 and Weri-Rb1 cells compared with cells transfected with mimics NC (Fig. 4a, b). In combination with previous results, we found that miR-491-3p inhibits Rb cell proliferation, colony formation and enhances cell apoptosis. Our study also indicated that EMT process was reversed when miR-491-3p is overexpressed. As shown in Fig. 4c, d, E-cadherin was significantly increased, while $\mathrm{N}$-cadherin and Vimentin were significantly decreased after Rb cells was transfected with miR-491-3p mimics. Our ICC results were also aligned with the result from western blotting, which E-cadherin was seen increased but both $\mathrm{N}$-cadherin and Vimentin were significantly decreased in miR-491-3p mimics group as compared to

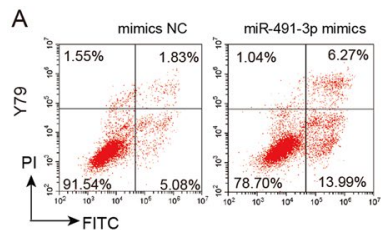

C

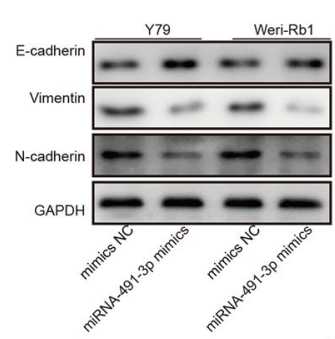

$E$
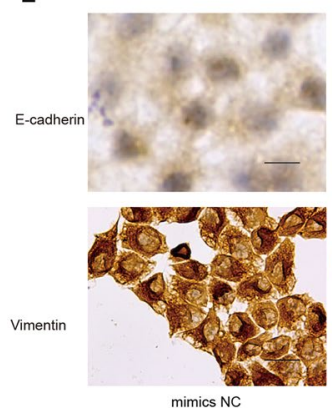

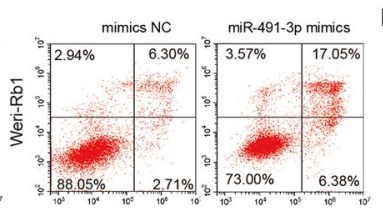

D



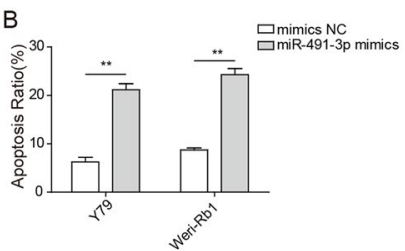

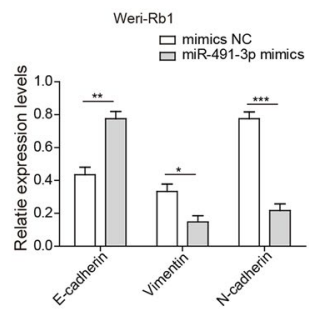

Y79

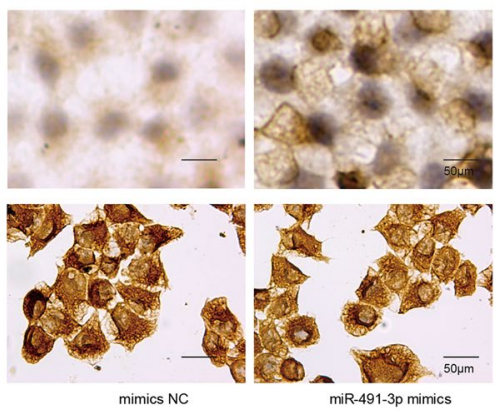

Fig. 4 Overexpression of miR-491-3p induced apoptosis and inhibited EMT in Rb cell lines. a FACS assay of apoptosis in Y79 and Weri-Rb1 cells transfected with miR-491-3p mimics, mimics NC, NC. b Apoptosis ratio was quantitatively analyzed. All results are from three independent experiments. c, d After Rb cell line transfected with miR-491-3p mimics, mimics NC, E-cadherin protein level was increased, while vimentin and $\mathrm{N}$-cadherin level were decreased compared to cells transfected with mimics NC. e In situ immunocytochemistry of Rb cell line transfected with miR-491-3p mimics, mimics NC (magnification, $\times 400$ ). Left image showed the morphology changes of cells, right image is statistical analysis of left image. All results are from three independent experiments. Bars represent mean \pm SD $(n=3, * p<0.05)$ 
miR-491-3p NC group. Morphology changes were noticeable in Y79 and Weri-Rb1 cells, too, both cell cultures cohesion had decreased and became more adhering in miR-491-3p mimics group as compared to miR-491-3p NC group which was more cohesive and spheroidal (Fig. 4e). The results suggested that overexpression of miR491-3p inhibited migration and invasion of $\mathrm{Rb}$ cells through regulating EMT.

\section{Low Expression of miR-491-3p Inhibits Apoptosis and Enhances the EMT Process of ARPE-19 Cells}

To further verify the effect of miR-491-3p on retinoblastoma. Another piece of data to include miR-491-3p inhibitor and inhibitor $\mathrm{NC}$ in the normal cells were conducted. ARPE-19 cells transfected with miR-491-3p inhibitor had lower level of miR-491-3p expression than inhibitor NC (Fig. S1A). Further, MTS assay results demonstrated that proliferation in ARPE-19 cells from 24 to $72 \mathrm{~h}$ had increased when ARPE-19 cells were transfected with miR-491-3p inhibitor as compared to cells transfected with inhibitor NC (Fig. S1B). We found that cells transfected with miR-491-3p inhibitors showed further decreased in cell apoptosis as compared to inhibitor NC (Fig. S1C). As shown in Fig. S1D, E-cadherin was significantly decreased, while $\mathrm{N}$-cadherin and Vimentin were significantly increased after $\mathrm{Rb}$ cells was transfected with miR-491-3p inhibitor as compared to the cells transfected with inhibitor NC. In combination with previous results, we found that low expression of miR-491-3p inhibits apoptosis and enhances the EMT process of ARPE-19 cells.

\section{Stannin is Targeted by miR-491-3p and Upregulated In Vivo and In Vitro}

Using four publicly available miRNA databases Hoctar, miRDB, mirDIP and Targetscan, we predicted that miR-491-3p pairs with mRNA Stannin (SNN) at position 2808-2815. Between 127 and 131 genes came out as results after we applied our prediction criteria to each of these prediction tool, but only 1 gene, SNN was shared by all the results of these tools (Fig. 5a, b). The results from our luciferase reporter assay have confirmed that SNN-WT interacted with miR-491-3p mimics as the activity of the SNN-WT in both Y79 and Weri-Rb1 cells were significantly reduced in the present of miR-491-3p mimics as compared to mimics NC. The result also showed that miR-491-3p did not affect SNN-MUT. This result indicated that miR491-3p only interacted with wild-type SNN by binding to the specific site of the gene, and miR-491-3p has no effect on SNN-MUT as the binding site was mutated (Fig. 5c). Our western blot results also demonstrated that SNN was downregulated with the overexpression of miR-493-3p, but was upregulated when miR-493-3p was suppressed (Fig. 5d). RT-qPCR, our study further revealed that SNN was overexpressed in the both Rb cells Weri-Rb1 and Y79 as compared to normal and ARPE19 cells (Fig. 5e, f). We were also able to confirm that transfection rate of SNN to the Weri-Rb1 and Y79 was very high as the SNN expression was more than tenfold higher as compare to the vector group (Fig. $5 \mathrm{~g}$ ). The results indicated that miR491-3p targeted and regulated SNN in both Weri-Rb1 and Y79 cells. 
A



B
Position 2808-2815 5'-AAUUUCAAUAAAAUUUGCAUAAA-3'
of SNN 3'UTR

hsa-miR-491-3p 3'-CAUCUUCCCUUAGAACGUAUUC-5'

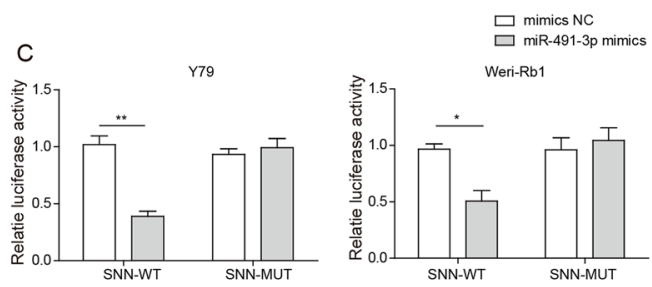

D
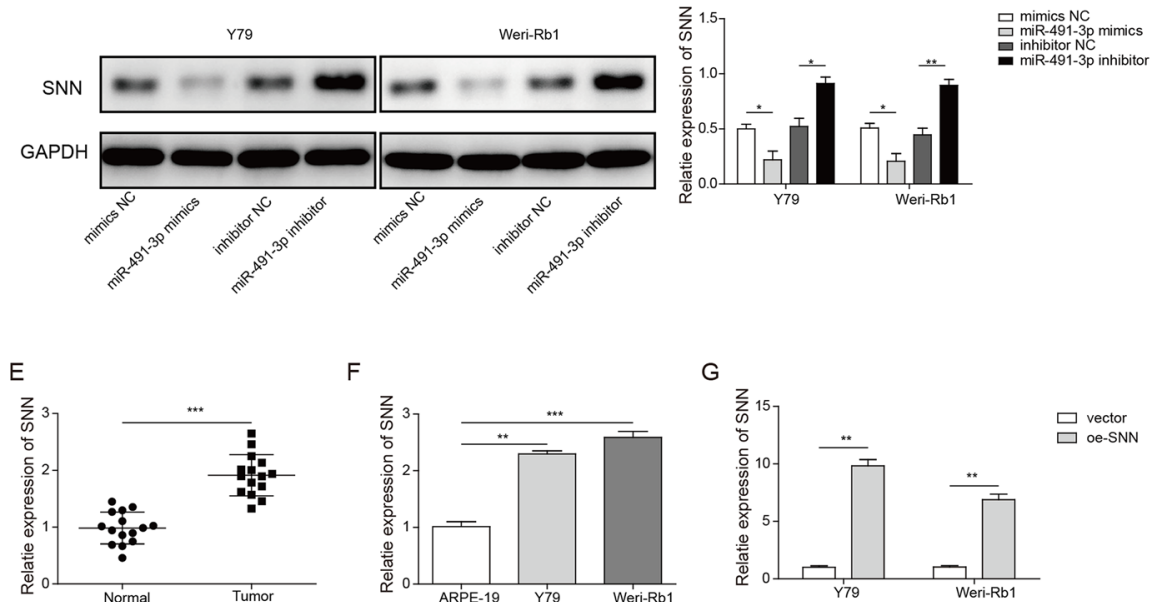

$\mathrm{F}$

G
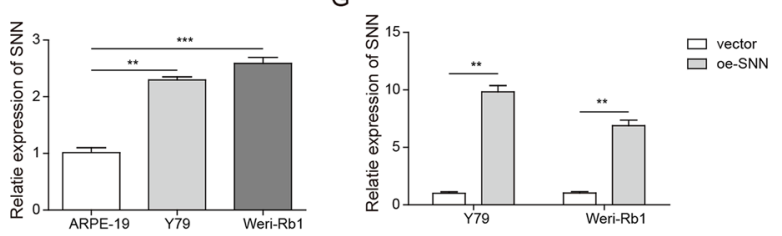

Fig. 5 Stannin is targeted by miR-491-3p and upregulated in vivo and in vitro. a The prediction result was taken from the intersection of the 4 miRNA target prediction databases. b Prediction of pairing location of miRNA miR-491-3p to mRNA SNN. c Luciferase reporter assay of Y79 or Weri-Rb1 cells with SNN-WT or SNN-MUT transfected with mimic NC or miR-491-3p mimics. All results are from three independent experiments. d Western blotting of the expression of SNN in Y79 and Weri-Rb1 cells transfected with mimics NC, miR-491-3p mimics. e RT-qPCR analysis of expression of SNN in Rb tissues and adjacent noncancerous tissues. All results are from three independent experiments. f RT-qPCR analysis of expression of SNN in ARPE-19, Weri-Rb1 or Y79 cells. All results are from three independent experiments. g RT-qPCR analysis of transfection rate of oe-SNN in the Y79 or Weri-Rb1 cells. All results are from three independent experiments. Bars represent mean $\pm \mathrm{SD}(n=3, * p<0.05)$

\section{SNN Eliminates Promotion of miR-491-3p on Rb Cells Proliferation, Migration and Invasion}

To understand the relationship of SNN to miR-491-3p in Rb, Y79 and Weri-Rb1 cells were transfected with either mimics NC, miR-491-3p mimics, miR-491-3p mimics + vector or miR-491-3p mimics + oe-SNN; subsequently, the proliferation activities were measured using MTS and colony formation assays. The results demonstrated that overexpression of the SNN cancel out the cell proliferation 
suppression effects of the miR-491-3p mimics in Weri-Rb1 and Y79 cells from 24 to $72 \mathrm{~h}$ (Fig. 6a). The soft agar colony formation assay showed that colonies of both $\mathrm{Rb}$ cell lines were significantly inhibited after miR-491-3p mimics transfection, but then


$\mathrm{B}$

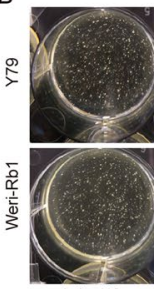

mimics NC

C

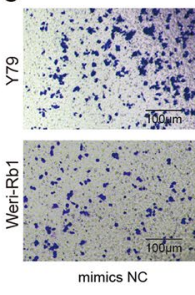

miR-491-3p mimics

mimics+vector

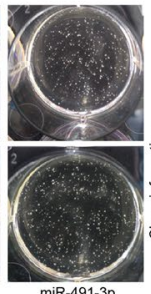

miR-491-3p
mimics+oe-SNN

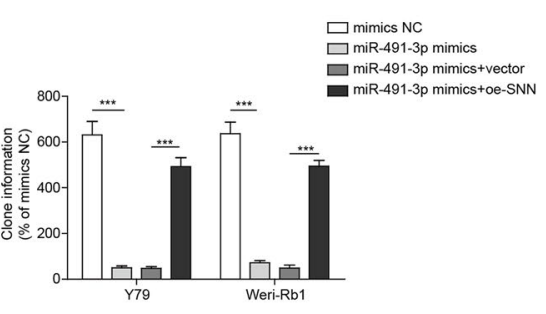

Y79


D
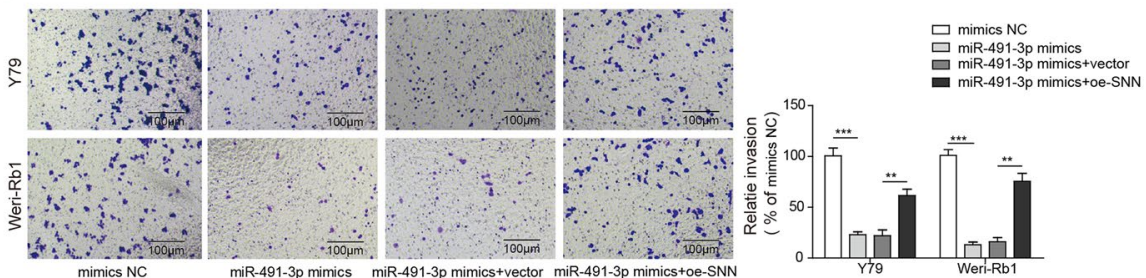

Fig. $6 \mathrm{SNN}$ eliminates promotion of miR-491-3p on $\mathrm{Rb}$ cells proliferation, migration and invasion. a MTS assay of cell proliferation of Y79 and Weri-Rb1 cells, or transfected with miR-491-3p mimics, miR-491-3p + vector or miR-491-3p+oe-SNN. b Colony formation assay of cell proliferation in Y79 and Weri-Rb1 cells, or transfected with miR-491-3p mimics, miR-491-3p + vector or miR-491-3p + oeSNN. Left images are clone formation on the culture dish of $\mathrm{Y} 79$ and Weri-Rb1 cells, or transfected with miR-491-3p mimics, miR-491-3p + vector or miR-491-3p + oe-SNN; right chart is the number of clones quantitatively analyzed. All results are from three independent experiments. $\mathbf{c}$ Left images are staining of cell migration of Y79 and Weri-Rb1 cells, or transfected with miR-491-3p mimics, miR-491-3p + vector or miR-491-3p +oe-SNN under inverted microscope (magnification, $\times 100$ ); right chart is statistical analysis of these clone cultures. $\mathbf{d}$ Left images are staining of cell invasion of Y79 and Weri-Rb1 cells, or transfected with miR-491-3p mimics, miR-491-3p + vector or miR-491-3p+oe-SNN under inverted microscope (magnification, $\times 100$ ); right chart is statistical analysis of these clone cultures. All results are from three independent experiments. Bars represent mean $\pm \mathrm{SD}\left(n=3,{ }^{*} p<0.05\right)$ 
elevated by the overexpression of SNN (Fig. 6b). The role of SNN in cell migration and invasion were also examined with transwell assays. The migrated $\mathrm{Rb}$ cells were decrease significantly after transfected with miR-491-3p mimics were subsequently promoted by the overexpression of SNN. The effect of miR-491-3p mimics was almost canceled out entirely by oe-SNN (Fig. 6c, d). Our result showed that SNN eliminated the effect of proliferation, migration and invasion those promoted by miR-491-3p in Rb cells.

\section{SNN Eliminates Influence of miR-491-3p on Apoptosis and EMT of Rb Cells}

To examine effect of SNN in Rb cell apoptosis and its involvement in the EMT process, we performed FACS assay and western blotting in present study. The results showed that overexpression of the SNN inhibited the enhancement of the miR491-3p mimics made to the $\mathrm{Rb}$ cells apoptosis. The apoptosis rates of both WeriRb1 and Y79 cells were similar to mimics NC groups when miR-491-3p mimics were co-transfected with oe-SNN (Fig. 7a). Western blotting's results showed similar trend, E-cadherin protein expression was significantly increased with the present of miR-491-3p mimics, but subsequently decreased after oe-SNN was transfected to the Rb cells. Vice versa, $\mathrm{N}$-cadherin and Vimentin expressions were significantly decreased by miR-491-3p mimics, and subsequently increased with the transfection of oe-SNN (Fig. 7b). Our ICC result also confirmed similar trends, where protein expression of E-cadherin was increased, while N-cadherin and Vimentin's expression were decreased with the present of miR-491-3p mimics, and oe-SNN reversed the effects. Cells cultures morphology changes were noticeable, too, cell cohesion decreased and cell became adhering in miR-491-3p mimics group, while cells were more cohesive and spheroidal in oe-SNN group (Fig. 7c). The results implied that the EMT process was inhibited by miR-491-3p but restored by the overexpression of $\mathrm{SNN}$ in the Rb development.

\section{Discussion}

$\mathrm{Rb}$ is the deadliest intraocular malignant tumor in the retina of children and its prevalence has increased worldwide. Numerous studies showed that miRNAs play critical roles in tumor pathogenesis (Gao et al. 2017; Sethi et al. 2014b). Therefore, miRNAs are considered to be effective diagnostic and prognostic biomarkers or therapeutic target. Many chemical drugs exert their therapeutic affects through regulating and targeting various miRNAs in tumor (Salarinia et al. 2016). Additionally, downregulation of miRNAs are often associated with progression of tumor cells (Busch et al. 2017; Samal et al. 2017). Recently, many researchers demonstrated that miR-491-3p is downregulated in different tumors, and functions as a tumor inhibitor in the tumorigenesis of several malignancies by affecting multiple genes (Duan et al. 2017; Zhao et al. 2017; Zheng et al. 2015). However, prior to the present study, the role of miR-491-3p in $\mathrm{Rb}$ remains unclear. In the present study, we showed that overexpression miR-491-3p in Rb cells 

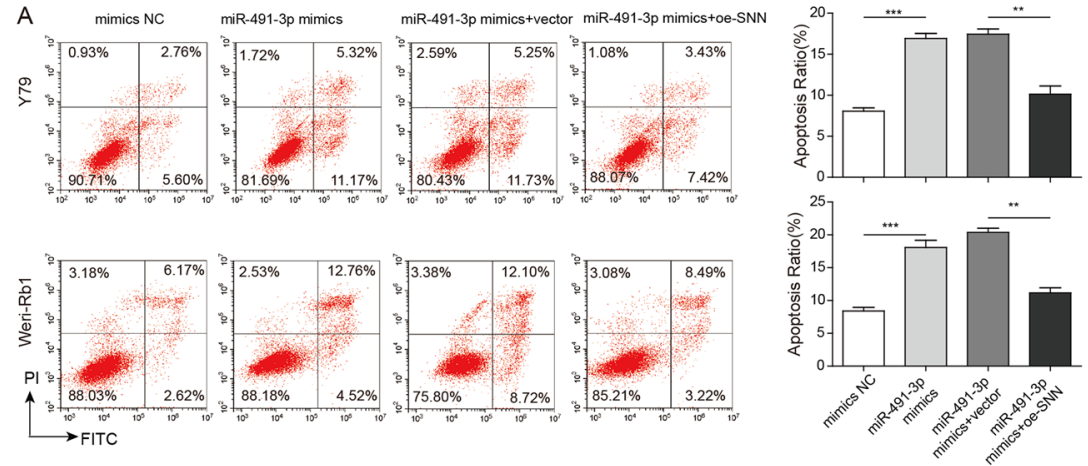

B

\section{$\square$ mimics NC}

$\square$ miR-491-3p mimics

$\square$ miR-491-3p mimics+vector

- miR-491-3p mimics+oe-SNN

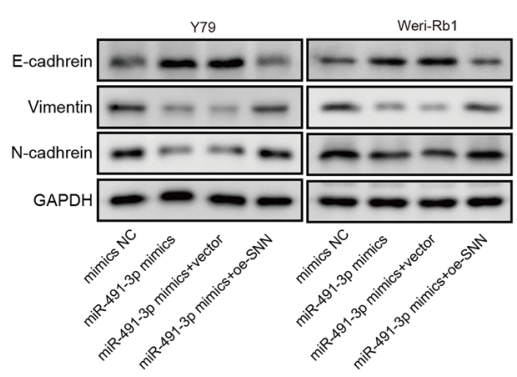
Weri-Rb1

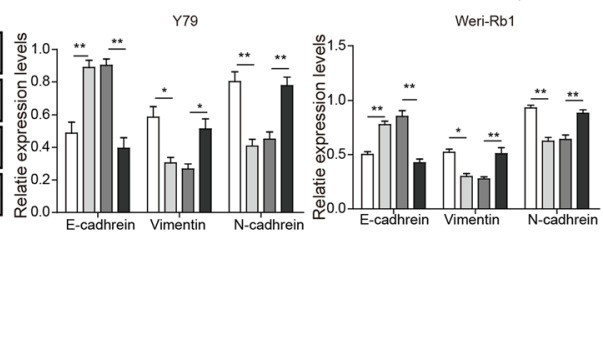

C



Fig. 7 SNN eliminates influence of miR-491-3p on apoptosis and EMT of Rb cells. a Left images are the FACS assay of apoptosis in Y79 and Weri-Rb1 cells, or transfected with miR-491-3p mimics, miR491-3p + vector or miR-491-3p+oe-SNN. Right chart is the quantitative analysis of the apoptosis rate of these images. b E-cadherin, vimentin and N-cadherin protein levels in Y79 and Weri-Rb1 cells, or transfected with miR-491-3p mimics, miR-491-3p+vector or miR-491-3p + oe-SNN. c In situ immunocytochemistry of $\mathrm{Rb}$ cell line transfected with miR-491-3p mimics, miR-491-3p + vector or miR491-3p + oe-SNN (magnification, $\times 400$ ). Left image showed the morphology changes of cells, right image is statistical analysis of left image. All results are from three independent experiments. Bars represent mean $\pm \operatorname{SD}(n=3, * p<0.05)$

can suppress proliferation and induce apoptosis as well as promote cell migration and invasion. In additional, we also found that epithelial marker E-cadherin expression was increased, while mesenchymal markers $\mathrm{N}$-cadherin and Vimentin expressions were decreased, cells became less cohesive and more adhering after miR-491-3p overexpression, suggesting EMT in Rb cells was regulated by miR-491-3p. 
Many researchers have reported that the expression of miRNAs were downregulated in various tumor tissues (Drusco and Croce 2017; Han Li and Chen 2015). While in $\mathrm{Rb}$, expressions of a number of miRNAs such as miRs-382, -486-3p, -129$3 p$, and $-129-5 p$ were suppressed in primary human Rb tissues and cell lines (Golabchi et al. 2018; Singh et al. 2016). It was identified that miR-491-3p expression was frequently decreased in osteosarcoma tissues and osteosarcoma cell lines, which functions as a tumor suppressor to attenuate the potential of growth and invasion by targeting TSPAN1 (Duan et al. 2017). The present study consistently demonstrates miR-491-3p expression is significantly downregulated in Rb tissues and $\mathrm{Rb}$ cell lines when compared with adjacent noncancerous tissues and ARPE-19 cells.

Extensive evidence has shown that overexpression of certain miRNAs can suppress tumor cell proliferation and enhances cell apoptosis in nearly all cancer types, such as skin cancer, lung cancer and cervical cancer (Li et al. 2018a; Ross et al. 2017; Vannini et al. 2018). Similarly, miRNAs also play important role in Rb. For example, miR-21 inhibited the cell proliferation via the modulation the cell apoptotic in Rb (Gui et al. 2016; Song et al. 2017). More importantly, miR-491-3p was also demonstrated to modulate tumor cell apoptosis and proliferation. Duan et al. found that as a tumor suppressor, restored miR-491-3p expression suppressed the growth of osteosarcoma cells (Duan et al. 2017). miR-491-3p also involved in tongue cancer and hepatocellular carcinoma (Zhao et al. 2017; Zheng et al. 2015). In this study, our findings showed that overexpression of miR-491-3p induces $\mathrm{Rb}$ cell apoptosis, and subsequently inhibits cell proliferation are consistent previous reports listed above. The results suggest that miR-491-3p is a tumor suppressor in $\mathrm{Rb}$.

Additionally, we also showed that miR-491-3p can inhibit cell migration, invasion and EMT in Rb cells and tissues. EMT is a crucial step in cancer invasion and metastasis, in which cells lose their epithelial characteristics, and are replaced by a mesenchymal phenotype such as reduced cell-cell adhesion as well as increased migratory and invasive properties (Shao et al. 2017; Thiery 2002). In details, we found that E-cadherin expression was increased, while vimentin and $\mathrm{N}$-cadherin expressions were decreased after overexpressing miR-491-3p in $\mathrm{Rb}$ cells. Epithelial marker E-cadherin is a type of $\mathrm{Ca}^{2+}$-dependent transmembrane glycoproteins regulates cell-cell adhesion. A deficiency of E-cadherin reduces the cell adhesion and promotes the cell migration and invasion in tumors. E-cadherin is also correlated with the prognosis of $\mathrm{Rb}$, lower E-cadherin generally indicates bad prognosis (Shao et al. 2017). Vimentin and N-cadherin are mesenchymal markers indicating the initiation of cancer metastasis. Consistence to our results, a number of studies showed that miRNAs may regulate EMT in a variety of cancers. A group of researchers reported that miR-302b inhibits tumorigenesis by targeting EphA2 via EMT signaling cascade in gastric cancer (Huang et al. 2017), and others found that miR-125b-5p can function as a tumor suppressor in esophageal squamous cell carcinoma (Mei et al. 2017). In the present study, our data confirmed that miR-491-3p could inhibit migration and invasion in Rb cells through regulating EMT, and it may be a novel targeted therapy for Rb. According to published research, miR-491-3p directly targeted insulin-like growth factor 1 receptor (IGF1R) and MKK7, a mitogen-activated protein kinase, to suppress tumor metastasis (Kumar et al. 2017; Sakai et al. 2014). MiR-491-3p was also 
reported to directly target RhoC and FZD4 to suppress bladder tumor cell mobility (Ueno et al. 2012). Finally miR-491-3p was reported to target YTHDF2, a methylation of the N6 position of adenosine (m6A) reader protein, and mitotic arrest deficient-2 (Mad2) to affect tumor cell proliferation (Li et al. 2018b; Tambe et al. 2016). We believe that they work similarly in Rb tumor genesis and progression. In future study, more experiments will be conducted in order to explore the interaction among miR-491-3p, YTHDF2 and Mad2 in tumor suppression in Rb.

The present study found out the miR-491-3p targeted mRNA SNN. Target sitemutated SNN did not interact with miR-491-3p in our study has confirmed our prediction, which was consistence with similar approach in other studies (Cheng et al. 2019; Dong et al. 2020). But there was a pity that we were unable to include SSN-MUT in the rest of our experiments certainly was an unfortunate limitation to our study that was due to time and cost constraint, but we will certainly revisit this limitation in our future study. SNN has been shown to play an important role in the toxic effect of organotin (Davidson et al. 2004) and endosomal maturation (Pueyo et al. 2016). Study of SNN in oncology, however, is scarce, but Reese et al. demonstrated that protein kinase $\mathrm{C}$ regulates Tumor Necrosis Factor-Alpha (TNF- $\alpha$ ) induced SNN expression (Reese et al. 2005), and our study is the first to reveal miR-491-3p and SNN's interaction to regulate proliferation, migration, invasion and apoptosis of Rb cell. We also know that SNN is highly involved in MAPK signaling pathways (Davidson et al. 2005) and MAPK signaling pathway has shown to be important in some cancers (Dhillon et al. 2007). This is consistence to our study which showed the proliferation, migration and invasion of the $\mathrm{Rb}$ cells correspond to the over expression of SNN, and it silences the effects of miR-491-3p. Our study has showed that overexpression of SNN promotes EMT process, too. The results suggested that SNN may be linked to cell growth and proliferation by regulating EMT process via MAPK signaling pathway. Summarizing all the above studies, we speculate miR-491-3p-SNN link may have effect in other tumors, but need further investigation. However, the mechanism of the mRNA SNN's involvement in the promotion of the Rb cells was not included in the present study. We do hope the present study may provide a renew interest for the future research.

In conclusion, miR-491-3p was markedly downregulated in $\mathrm{Rb}$ tissues and $\mathrm{Rb}$ cells. It inhibited tumor growth by inducing cell apoptosis, and suppressed the cell proliferation, cell migration and invasion of $\mathrm{Rb}$ cells. In additional, miR-491-3p dramatically increased the expression of E-cadherin and decreased the expression of Vimentin and $\mathrm{N}$-cadherin in Rb cell lines, suggesting that it was involved in EMT of Rbs. Our study will provide novel insights into miR-491-3p as a tumor suppressor in $\mathrm{Rb}$, and it could be a therapeutic target for $\mathrm{Rb}$. However, the underlying mechanism of how miR-491-3p regulates Rb cell EMT markers and other bioactivities is still dimness. Thus, further study is needed to elucidate the entire cellular and molecular pathway of miR-491-3p in Rb. Additionally, we also planned in vivo animal experiments to confirm our in vitro results in future.

Acknowledgements We would like to give our sincere gratitude to the reviewers for their constructive comments. 
Author Contributions Guarantor of integrity of the entire study: Y.H.; study concepts: Y.H.; study design: Y.H.; definition of intellectual content: Y.H.; literature research: Y.H., M.Z., L.L.; clinical studies: Y.H., J.D., Y-M.G., T-W.W.; experimental studies: Y.H., J.D., Y-M.G., T-W.W.; data acquisition: Y-M.G., T-W.W.; data analysis: Y.H., Y-M.G., T-W.W.; statistical analysis: Y.H., Y-M.G., T-W.W.; manuscript preparation: Y.H., Y-M.G.; manuscript editing: Y.H., T-W.W.; manuscript review: Y.H., M.Z.

Funding This research did not receive any specific grant from funding agencies in the public, commercial, or not-for-profit sectors.

Data Availability All statistical analyses were performed using SPSS 18.0 software (SPSS Inc., Chicago, IL, USA).

\section{Compliance with Ethical Standards}

Conflict of interest The authors declare that they have no conflict of interests.

Ethical Approval All experiments were preformed according to Wuhan University of Science and Technology's human object use guidelines with approval from IRB (institution review board) and Puren Hospital of Wuhan University of Science and Technology's ethics committee.

Informed Consent The consent to participate was obtained from patients.

Consent for Publication The informed consent obtained from study participants.

Open Access This article is licensed under a Creative Commons Attribution 4.0 International License, which permits use, sharing, adaptation, distribution and reproduction in any medium or format, as long as you give appropriate credit to the original author(s) and the source, provide a link to the Creative Commons licence, and indicate if changes were made. The images or other third party material in this article are included in the article's Creative Commons licence, unless indicated otherwise in a credit line to the material. If material is not included in the article's Creative Commons licence and your intended use is not permitted by statutory regulation or exceeds the permitted use, you will need to obtain permission directly from the copyright holder. To view a copy of this licence, visit http://creativecommons.org/licen ses/by/4.0/.

\section{References}

Busch M et al (2017) Reduction of the tumorigenic potential of human retinoblastoma cell lines by TFF1 overexpression involves p53/caspase signaling and miR-18a regulation. Int J Cancer 141:549-560. https://doi.org/10.1002/ijc.30768

Cheng Y, Chang Q, Zheng B, Xu J, Li H, Wang R (2019) LncRNA XIST promotes the epithelial to mesenchymal transition of retinoblastoma via sponging miR-101. Eur J Pharmacol 843:210-216. https ://doi.org/10.1016/j.ejphar.2018.11.028

Davidson CE, Reese BE, Billingsley ML, Yun JK (2004) Stannin, a protein that localizes to the mitochondria and sensitizes NIH-3T3 cells to trimethyltin and dimethyltin toxicity. Mol Pharmacol 66:855-863. https://doi.org/10.1124/mol.104.001719

Davidson CE, Reese BE, Billingsley ML, Yun JK (2005) The protein stannin binds 14-3-3zeta and modulates mitogen-activated protein kinase signaling. Brain Res Mol Brain Res 138:256-263. https:// doi.org/10.1016/j.molbrainres.2005.04.018

Dhillon AS, Hagan S, Rath O, Kolch W (2007) MAP kinase signalling pathways in cancer. Oncogene 26:3279-3290. https://doi.org/10.1038/sj.onc.1210421

Dong Y, Wan G, Yan P, Qian C, Li F, Peng G (2020) Long noncoding RNA LINC00324 promotes retinoblastoma progression by acting as a competing endogenous RNA for microRNA-769-5p, thereby 
increasing STAT3 expression. Aging (Albany NY) 12:7729-7746. https://doi.org/10.18632/aging .103075

Drusco A, Croce CM (2017) MicroRNAs and cancer: a long story for short RNAs. Adv Cancer Res 135:1-24. https://doi.org/10.1016/bs.acr.2017.06.005

Duan J, Liu J, Liu Y, Huang B, Rao L (2017) miR-491-3p suppresses the growth and invasion of osteosarcoma cells by targeting TSPAN1. Mol Med Rep 16:5568-5574. https://doi.org/10.3892/ mmr.2017.7256

Fernandes AG, Pollock BD, Rabito FA (2017) Retinoblastoma in the United States: a 40-year incidence and survival analysis. J Pediatr Ophthalmol Strabismus. https://doi.org/10.3928/01913913-20171 116-03

Gao Y, Lin L, Li T, Yang J, Wei Y (2017) The role of miRNA-223 in cancer: function, diagnosis and therapy. Gene 616:1-7. https://doi.org/10.1016/j.gene.2017.03.021

Gennarino VA, Sardiello M, Mutarelli M, Dharmalingam G, Maselli V, Lago G, Banfi S (2011) HOCTAR database: a unique resource for microRNA target prediction. Gene 480:51-58. https://doi. org/10.1016/j.gene.2011.03.005

Golabchi K et al (2018) MicroRNAs in retinoblastoma: potential diagnostic and therapeutic biomarkers. J Cell Physiol 233:3016-3023. https://doi.org/10.1002/jcp.26070

Gui F, Hong Z, You Z, Wu H, Zhang Y (2016) MiR-21 inhibitor suppressed the progression of retinoblastoma via the modulation of PTEN/PI3K/AKT pathway. Cell Biol Int 40:1294-1302. https://doi. org/10.1002/cbin.10678

Han Li C, Chen Y (2015) Small and long non-coding RNAs: novel targets in perspective cancer therapy. Curr Genom 16:319-326. https://doi.org/10.2174/1389202916666150707155851

Huang J et al (2017) miR-302b inhibits tumorigenesis by targeting EphA2 via Wnt/ beta-catenin/ EMT signaling cascade in gastric cancer. BMC Cancer 17:886. https://doi.org/10.1186/s1288 5-017-3875-3

Kumar AS, Jagadeeshan S, Pitani RS, Ramshankar V, Venkitasamy K, Venkatraman G, Rayala SK (2017) Snail-modulated MICRORNA 493 forms a negative feedback loop with the insulin-like growth factor 1 receptor pathway and blocks tumorigenesis. Mol Cell Biol. https://doi.org/10.1128/ MCB.00510-16

Li GC, Cao XY, Li YN, Qiu YY, Li YN, Liu XJ, Sun XX (2018) MicroRNA-374b inhibits cervical cancer cell proliferation and induces apoptosis through the p38/ERK signaling pathway by binding to JAM-2. J Cell Physiol. https://doi.org/10.1002/jcp.26574

Li J et al (2018) Downregulation of N(6)-methyladenosine binding YTHDF2 protein mediated by miR493-3p suppresses prostate cancer by elevating N(6)-methyladenosine levels. Oncotarget 9:37523764. https://doi.org/10.18632/oncotarget.23365

Ma Q, Song J, Chen Z, Li L, Wang B, He N (2018) Identification of potential gene and microRNA biomarkers for colon cancer by an integrated bioinformatical approach. Trans Cancer Res 7:17-29

Mei LL, Wang WJ, Qiu YT, Xie XF, Bai J, Shi ZZ (2017) miR-125b-5p functions as a tumor suppressor gene partially by regulating HMGA2 in esophageal squamous cell carcinoma. PLoS ONE 12:e0185636. https://doi.org/10.1371/journal.pone.0185636

Neoplasms of the Eye (2003) In: Society AC (ed) Cancer Medicine. BC Decker Inc, Hamilton

Pueyo JI, Magny EG, Sampson CJ, Amin U, Evans IR, Bishop SA, Couso JP (2016) Hemotin, a regulator of phagocytosis encoded by a small ORF and conserved across metazoans. PLoS Biol 14:e1002395. https://doi.org/10.1371/journal.pbio.1002395

Ramirez-Ortiz MA et al (2017) Systematic review of the current status of programs and general knowledge of diagnosis and management of retinoblastoma. Bol Med Hosp Infant Mexico 74:41-54. https ://doi.org/10.1016/j.bmhimx.2016.08.002

Reese BE, Davidson C, Billingsley ML, Yun J (2005) Protein kinase C epsilon regulates tumor necrosis factor-alpha-induced stannin gene expression. J Pharmacol Exp Ther 314:61-69. https://doi. org/10.1124/jpet.105.084236

Reese BE, Krissinger D, Yun JK, Billingsley ML (2006) Elucidation of stannin function using microarray analysis: implications for cell cycle control. Gene Expr 13:41-52. https://doi.org/10.3727/00000 0006783991944

Ross CL, Kaushik S, Valdes-Rodriguez R, Anvekar R (2017) MicroRNAs in cutaneous melanoma: Role as diagnostic and prognostic biomarkers. J Cell Physiol. https://doi.org/10.1002/jcp.26395

Sakai H et al (2014) MKK7 mediates miR-493-dependent suppression of liver metastasis of colon cancer cells. Cancer Sci 105:425-430. https://doi.org/10.1111/cas.12380 
Salarinia R et al (2016) Epi-drugs and epi-mirs: moving beyond current cancer therapies. Curr Cancer Drug Targets 16:773-788

Samal J, Kandpal M, Vivekanandan P (2017) HBeAg-induced miR-106b promotes cell growth by targeting the retinoblastoma gene. Sci Rep 7:14371. https://doi.org/10.1038/s41598-017-14652-x

Sethi S, Ali S, Sethi S, Sarkar FH (2014a) MicroRNAs in personalized cancer therapy. Clin Genet 86:6873. https://doi.org/10.1111/cge.12362

Sethi S, Ali S, Sethi S, Sarkar FH (2014b) MicroRNAs in personalized cancer therapy. Clin Genet 86:68-73

Shao XL, Chen Y, Gao L (2017) MiR-200c suppresses the migration of retinoblastoma cells by reversing epithelial mesenchymal transition. Int J Ophthalmol 10:1195

Singh U, Malik MA, Goswami S, Shukla S, Kaur J (2016) Epigenetic regulation of human retinoblastoma. Tumour Biol 37:14427-14441. https://doi.org/10.1007/s13277-016-5308-3

Song D, Diao J, Yang Y, Chen Y (2017) MicroRNA382 inhibits cell proliferation and invasion of retinoblastoma by targeting BDNFmediated PI3K/AKT signalling pathway. Mol Med Rep 16:6428-6436. https://doi.org/10.3892/mmr.2017.7396

Tambe M et al (2016) Novel Mad2-targeting miR-493-3p controls mitotic fidelity and cancer cells' sensitivity to paclitaxel. Oncotarget 7:12267-12285. https://doi.org/10.18632/oncotarget.7860

Thiery JP (2002) Epithelial-mesenchymal transitions in tumour progression. Nat Rev Cancer 2:442

Tokar T et al (2018) mirDIP 4.1-integrative database of human microRNA target predictions. Nucleic Acids Res 46:D360-D370. https://doi.org/10.1093/nar/gkx1144

Ueno K et al (2012) Tumor suppressor microRNA-493 decreases cell motility and migration ability in human bladder cancer cells by downregulating RhoC and FZD4. Mol Cancer Ther 11:244-253. https://doi.org/10.1158/1535-7163.MCT-11-0592

Vannini I, Fanini F, Fabbri M (2018) Emerging roles of microRNAs in cancer Current opinion in genetics \& development 48:128-133. https://doi.org/10.1016/j.gde.2018.01.001

Wong N, Wang X (2015) miRDB: an online resource for microRNA target prediction and functional annotations. Nucleic Acids Res 43:D146-152. https://doi.org/10.1093/nar/gku1104

$\mathrm{Xu} \mathrm{X}$ et al (2011) Microarray-based analysis: identification of hypoxia-regulated microRNAs in retinoblastoma cells. Int J Oncol 38:1385-1393. https://doi.org/10.3892/ijo.2011.961

Zeisberg M, Neilson EG (2009) Biomarkers for epithelial-mesenchymal transitions. J Clin Investig 119:1429-1437. https://doi.org/10.1172/JCI36183

Zhao Y et al (2017) The miR-491-3p/Sp3/ABCB1 axis attenuates multidrug resistance of hepatocellular carcinoma. Cancer Lett 408:102-111. https://doi.org/10.1016/j.canlet.2017.08.027

Zheng G et al (2015) The miR-491-3p/mTORC2/FOXO1 regulatory loop modulates chemo-sensitivity in human tongue cancer. Oncotarget 6:6931-6943. https://doi.org/10.18632/oncotarget.3165

Zhu Y et al (2018) MiR-17-5p enhances pancreatic cancer proliferation by altering cell cycle profiles via disruption of RBL2/E2F4-repressing complexes. Cancer Lett 412:59-68. https://doi.org/10.1016/j. canlet.2017.09.044

Publisher's Note Springer Nature remains neutral with regard to jurisdictional claims in published maps and institutional affiliations.

\section{Affiliations}

\section{Yang Hu${ }^{1}$ (D) Ming Zhao ${ }^{1} \cdot \mathrm{Li} \mathrm{Li}^{1} \cdot$ Jie Ding ${ }^{1} \cdot$ Yu-Min Gui ${ }^{1} \cdot$ Tan-Wei Wei $^{1}$}

Yang $\mathrm{Hu}$

ophhy@163.com

1 Department of Ophthalmology, Puren Hospital of Wuhan University of Science and Technology, No.1 Benxi Road, Qingshan District, Wuhan 430080, Hubei Province, People's Republic of China 